\title{
Testing the Binary Black Hole Nature of a Compact Binary Coalescence
}

\author{
N. V. Krishnendu, ${ }^{1, *}$ K. G. Arun, ${ }^{1, \dagger}$ and Chandra Kant Mishra ${ }^{2,3, \neq}$ \\ ${ }^{1}$ Chennai Mathematical Institute, Siruseri, 603103, India. \\ ${ }^{2}$ Indian Institute of Technology Madras, Chennai - 600036, India. \\ ${ }^{3}$ ICTS-TIFR, Bengaluru (North) - 560089, India.
}

(Dated: September 7, 2017)

\begin{abstract}
We propose a novel method to test the binary black hole nature of compact binaries detectable by gravitational wave $(\mathrm{GW})$ interferometers and, hence, constrain the parameter space of other exotic compact objects. The spirit of the test lies in the "no-hair" conjecture for black holes where all properties of a Kerr black hole are characterized by its mass and spin. The method relies on observationally measuring the quadrupole moments of the compact binary constituents induced due to their spins. If the compact object is a Kerr black hole (BH), its quadrupole moment is expressible solely in terms of its mass and spin. Otherwise, the quadrupole moment can depend on additional parameters (such as the equation of state of the object). The higher order spin effects in phase and amplitude of a gravitational waveform, which explicitly contains the spin-induced quadrupole moments of compact objects, hence uniquely encode the nature of the compact binary. Thus, we argue that an independent measurement of the spin-induced quadrupole moment of the compact binaries from GW observations can provide a unique way to distinguish binary $\mathrm{BH}$ systems from binaries consisting of exotic compact objects.

PACS numbers: 04.30.Db, 04.25.Nx, 04.80.Nn, 95.55.Ym
\end{abstract}

Introduction: With the twin detections of binary black hole mergers by advanced LIGO interferometers [1], black holes (BHs) are no longer just elegant mathematical entities but a physical reality [2-4]. Now we know that BHs do exist in nature, and they can form a binary BH system and merge emitting gravitational waves (GWs) to form a single BH. Analytical frameworks of post-Newtonian theory ( $\mathrm{PN}$ ) [5] and BH perturbation theory [6] together with numerical relativity [7] have provided us a theoretical platform to study and interpret the GW observations of compact binary mergers. Both the observed events, GW150914 and GW151226, were found to be consistent with a binary black hole merger with approximate total masses of $65 M_{\odot}$ and $22 M_{\odot}$, respectively. The strong evidence for their binary black hole (BBH) nature comes from the following facts [8]: 1) Keplerian estimates of the orbital size are naturally explained by invoking a binary BH system, 2) the observed ringdown waveform is consistent with the least-damped quasinormal mode of a Kerr BH [9] (with the inferred final mass and spin), and finally, 3) the reconstructed signal matches excellently with the numerical relativity waveforms of a $\mathrm{BBH}$ merger.

With planned upgrades towards operating advanced LIGO detectors at respective design sensitivities and more detectors (such as advanced Virgo [10], KAGRA and LIGO-India [11]) joining the worldwide network of GW interferometers, many more such detections are likely to happen in the future observation runs [12]. One of the important questions from a fundamental physics viewpoint, is whether we can confidently distinguish the mergers of BBHs from that of binaries comprised of exotic compact objects such as gravastars [13] and boson stars [14], which may mimic many features of a BBH merger (see, also Ref.[15] for a recent review on possible $\mathrm{BH}$ mimickers and their $\mathrm{GW}$ signatures).

The definition of a Kerr BH is very closely tied with the the "no-hair" conjecture which says that all the properties of a Kerr BH are completely described by its mass and spin. The quasinormal mode spectrum of a Kerr BH that is formed, say, by the merger of two compact objects would, hence, be completely characterized by the mass and spin of the remnant $\mathrm{BH}$. This is a topic that has been studied in great detail over the past two decades. References [16,17] studied the abilities of GW detectors to carry out spectroscopy of a remnant compact object thereby testing its $\mathrm{BH}$ nature. The possibility of constraining specific $\mathrm{BH}$ mimicker models such as boson stars using quasinormal mode spectrum observations has been discussed in $[18,19]$. If we have a stellar mass $\mathrm{BH}$ orbiting a supermassive $\mathrm{BH}$ or an intermediate mass $\mathrm{BH}$ the dynamics of the stellar mass $\mathrm{BH}$ (treated as a test particle) would encode information about the multipole structure of the central $\mathrm{BH}$, and, therefore constrain any possible deviations from the $\mathrm{BH}$ nature [20-22]. While these methods are restricted to studying the $\mathrm{BH}$ nature of the central compact object, the recent proposals in Refs. [23-25] showed how the measurement of the tidal Love number of a compact binary may be used to detect exotic compact objects constituting a compact binary.

In this Letter, we propose a new method to test the binary black hole nature of the detected GW event by measuring the spin-induced quadrupole moments of the binary's constituents, whose values are unique for Kerr BHs in GR due to the no-hair conjecture. For an isolated Kerr BH, it is wellknown that the quadrupole moment scalar is given by $Q=-m^{3} \chi^{2}$, where $m$ is the mass of the $\mathrm{BH}$ and $\chi$ is the magnitude of the dimensionless spin parameter defined as $\vec{\chi}=\frac{\vec{S}}{m^{2}}$ (where $\vec{S}$ is the spin angular momentum vector of the $\mathrm{BH}$ ). For a non-BH compact object, this may be generalized to $Q=-\kappa m^{3} \chi^{2}$, with $\kappa=1$ as the $\mathrm{BH}$ limit. Depending on the equation of state, studies have shown that for neutron stars (NSs), $\kappa$ may range between $\sim 2$ and 14 [26, 27]), for boson stars between $\sim 10$ and 150 [28], and for (thin shell) gravastars, $\kappa$ may even take negative values [29] (which means the spin 

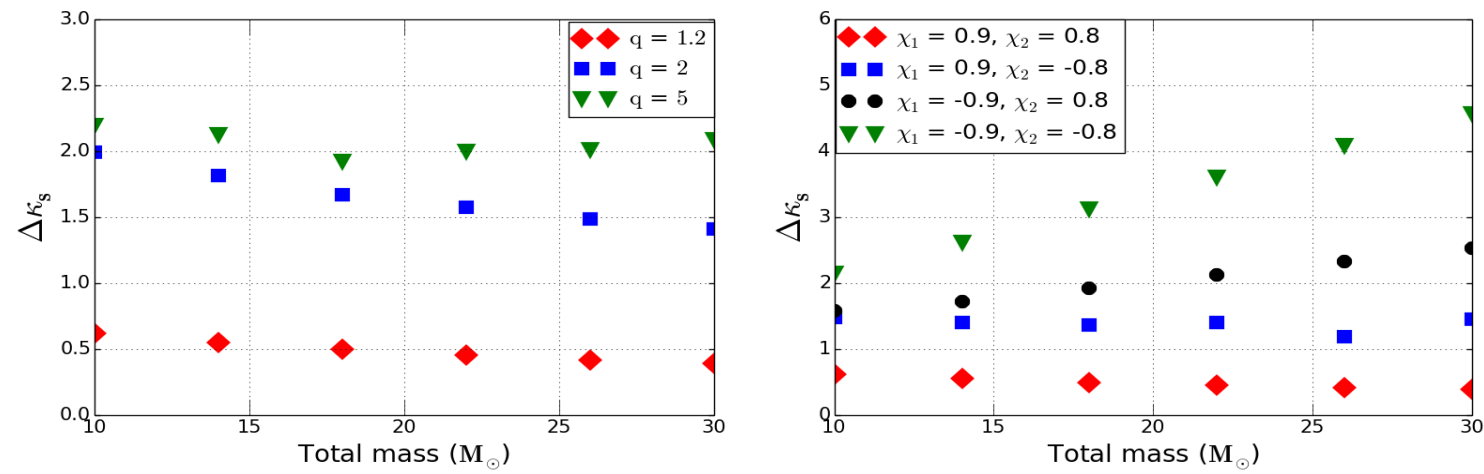

FIG. 1: Errors in measuring $\kappa_{s}$ as a function of the binary's total mass for three different mass ratio cases (top panel) and for different spin configurations (bottom panel) for advanced LIGO. The values of dimensionless spin parameters $\left(\chi_{1}, \chi_{2}\right)$ are fixed at 0.9 and 0.8 for the top panel plots, whereas mass ratio $(q)$ is fixed to be 1.2 for the plots in the bottom panel. Both panels assume a fixed inclination angle of the binary, $\iota=\frac{\pi}{3}$. The binary's location and other angular parameters are chosen in a way that produces an observed signal-to-noise ratio of 10.

leads to prolateness of the object instead of oblateness.)

In the PN model of compact binaries, the spin-induced quadrupole moment terms appear at the same order where the leading order quadratic-in-spin terms appear (note, $Q \propto \chi^{2}$ ), which is second PN order [30]. The parameter $\kappa$ that characterizes the magnitude of the spin-induced quadrupole moment (given the nature of the object) for each binary component can be tagged as $\kappa_{1}$ and $\kappa_{2}$ following the notation of Ref. [31] (throughout the paper, suffix 1 refers to the heavier compact binary component and 2 the lighter one). If we rewrite the waveforms in terms of the symmetric and antisymmetric combinations of $\kappa_{1}$ and $\kappa_{2}$ given by $\kappa_{s}=\left(\kappa_{1}+\kappa_{2}\right) / 2$ and $\kappa_{a}=\left(\kappa_{1}-\kappa_{2}\right) / 2$, respectively, then a BBH system is specified by $\kappa_{s}=1, \kappa_{a}=0$. This suggests, if we can accurately measure $\kappa_{s}$ and $\kappa_{a}$ to be 1 and 0 , respectively, we have established that the detected compact binary is a BBH.

However, note that $\kappa_{s}$ and $\kappa_{a}$ are highly degenerate parameters whose simultaneous extraction turns out to yield almost no constraint on them (this will have to be revisited using Bayesian methods in a future work). Hence, we resort to a method where we fix $\kappa_{a}$ to be 0 , as expected for a Kerr BBH, and then calculate the error bars associated with the measurement of $\kappa_{s}$ from GW observations. The aim here is to see how well can we estimate $\kappa_{s}$ around the true value of 1 (for a BBH) and, hence, confirm that the observed system is indeed a BBH. These error bars can be interpreted as upper bounds on the value of $\kappa_{s}$ allowed for exotic compact objects. In this sense, the proposed test is a "null test" of the BBH nature, where, observations would constrain the allowed range of deviations of $\kappa_{s}$ from the BBH value. Moreover, since the spirit of the test relies on the fact that quadrupole moments of BHs in a BBH system would depend only on the mass and the spin, the proposed test can be regarded as the no hair theorem test for the BBHs.

We wish to clarify that the error bars here refer to the width of the measured distribution of $\kappa_{s}$ at a fixed confidence level (in our case, $1-\sigma)$. Depending on the masses and spins of the system, this width may be much larger than 1 , in which case, this may be better interpreted as an upper bound on the allowed value of $\kappa_{s}$ for the given system. In most cases we have studied (in context of advanced LIGO), it is less than $\sim 20$ (see Figs. 1 and 2). Since $\kappa_{s}$ for interesting BH mimickers such as boson stars can be as high as 150 , the proposed method will be able to put stringent, model-independent constraints on the parameter space of $\mathrm{BH}$ mimickers. It should also be noted that though we have posed this as a null test, the proposed test can detect the signatures of exotic compact objects through a shift in the peak of the measured distribution away from 1, as is expected for BH mimickers.

In general, if we parametrize the deviation of $\kappa$ by $\kappa=1+\alpha$ (where $\alpha$ is the deformation parameter, which is 0 for BHs) and assume that the constituents of the binary are of identical types $\left(\alpha_{1}=\alpha_{2}\right)$, then, again, showing $\kappa_{s}=1$ is equivalent to showing the $\mathrm{BBH}$ nature of the compact binary system. This is because we again have $\kappa_{a} \equiv 0$, which is consistent with our original assumption for BBHs. Note that even if the detected compact binary constitutes two stars which have $\kappa \neq 1$, the proposed method will be sensitive in detecting them as they will add to the systematic offset in the measured value of $\kappa_{s}$ from 1 . Hence, our proposal to measure only $\kappa_{s}$ should work for compact binaries with any combination of compact objects when applied to the real data.

Waveform model. Because of the recent progresses in the post-Newtonian modeling of spinning compact binaries [31-35], we now have access to the higher order spin corrections to the GW phasing and amplitude. Here we use a waveform which is $2 \mathrm{PN}$ in amplitude and 4PN (note that the phasing formula at the 4PN only includes spin-orbit tail terms and hence is only partial. See a related discussion in Ref. [35]) in phase and spins of the two compact objects are considered to be 
along or opposite the orbital angular momentum vector of the binary. The spin-induced quadrupole moment coefficient appears at 2PN, 3PN and 3.5PN orders. The spin-induced octupole moment coefficient which appear at 3.5PN is set to 1, the BH value as we focus only on quadrupole here. See the section Supplemental Material below for details of the waveform model.

Estimation of $\kappa_{s}$ : We use the semianalytical parameter estimation technique based on the Fisher information matrix formalism [36] to deduce typical accuracies with which $\kappa_{s}$ may be estimated from GW observations. The Fisher information matrix approach allows us to calculate the widths of the posterior distribution of various parameters for Gaussian noise and in the limit of high signal-to noise-ratio (SNR) (see Ref. [37] for a detailed discussion on the possible caveats). Unlike previous works with PN waveforms which have subdominant modes (e.g. Refs. [38, 39]), we truncate the waveforms at $t$ wice the orbital frequency of the binary when it reaches the innermost stable circular orbit $\left(2 F_{\text {ISCO }}\right)$ as opposed to the choice of $k F_{\text {ISCO }}$, where $k$ is the maximum number of harmonics of the orbital phase present in the waveform. Here, the ISCO frequency is computed using numerical fitting formulas listed in Eqs. (3.7) and (3.8) of Refs. [40, 41]. By doing so, we hope to control the systematics due to the neglect of merger and ringdown. Though much less realistic than numerical methods based on algorithms such as Markov chain Monte Carlo (MCMC) calculations, the semianalytic method used here is significantly inexpensive in terms of computational time and is expected to match the predictions of numerical methods in the high SNR limit [42]. However, we caution that the errors we quote here should be taken as a typical order of magnitude of the expected errors which will be quantified in the future with MCMC investigations.
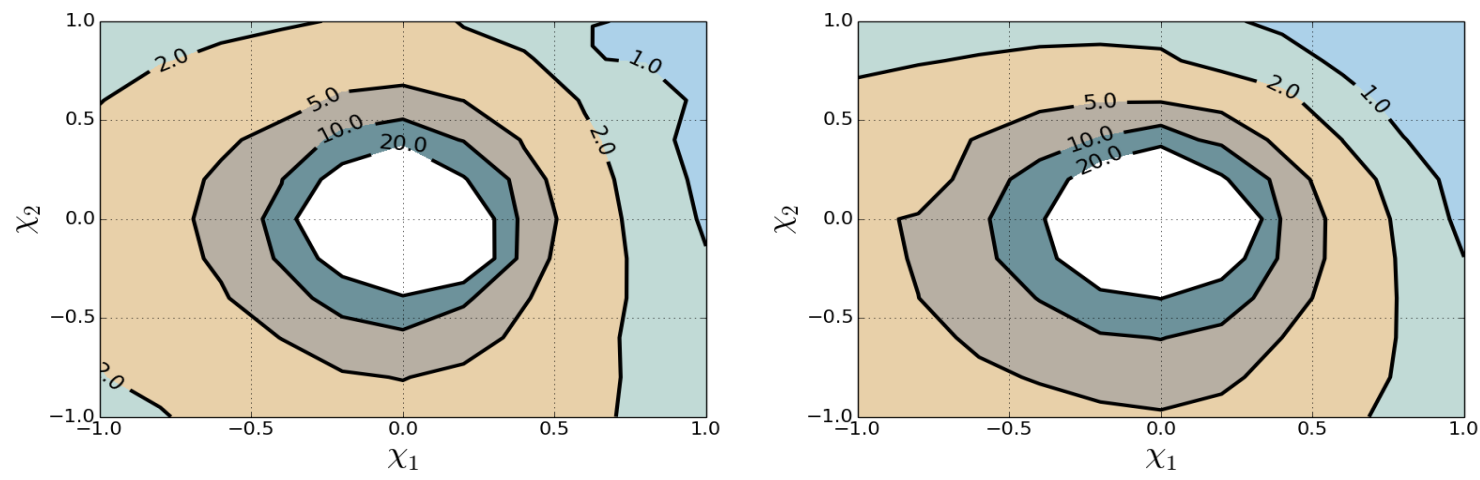

FIG. 2: Two-dimensional error contours indicating the measurability of $\kappa_{s}$ in the $\chi_{1}-\chi_{2}$ plane for two representative binary systems: $(5,4) M_{\odot}$ (top panel) and $(10,9) M_{\odot}$ (bottom panel) for advanced LIGO sensitivity. The inclination angle of the binary is chosen to a value of $\pi / 3$, and the source is located and oriented in such a way that it produces a signal-to-noise ratio of 10 at the detector.

For every system of interest, we construct a Fisher information matrix using the waveform model discussed above for the set of parameters $\left\{t_{c}, \phi_{c}, D_{L}, \iota, \mathcal{M}, \delta, \chi_{1}, \chi_{2}, \kappa_{s}\right\}$ which describe the signal. Here, $t_{c}$ and $\phi_{c}$ denote time and phase of the waveform at coalescence, two mass parameters $\mathcal{M}=\left(m_{1} m_{2}\right)^{3 / 5} /\left(m_{1}+m_{2}\right)^{2 / 5}$ and $\delta=\left|m_{1}-m_{2}\right| /\left(m_{1}+m_{2}\right)$ are known as the chirp mass and difference mass ratio of the binary, parameters $\left(\chi_{1}, \chi_{2}\right)$ denote the dimensionless spins of the binary components, and finally, $D_{L}$ and $\iota$ are the luminosity distance and the inclination angle of the binary, respectively. We consider the problem from a single detector standpoint and, hence, do not include the angles which describe the source location in the set of parameters. We compute the lower bound on the errors of each parameter (Cramer-Rao bound) by taking the square root of the diagonal values of the inverse of the $9 \times 9$ Fisher information matrix (covariance matrix). These errors are calculated for different masses and spins of the compact binary systems as well as for different inclination angles $(\iota)$. We consider the sources to be located and oriented in such a way that they produce a SNR of 10 at the detector. Projected advanced LIGO noise PSD [43] is used to compute the errors. The $1-\sigma$ error bars on $\kappa_{s}$ (with a peak at 1 ) assume $\kappa_{a}=0$, which is the case for Kerr BBHs. From a GW event, if we find that the posterior distribution for $\kappa_{s}$ is offset from 1, it may be taken as a signature for at least one of the binary components to be a non-BH object. Throughout the Letter, we quote errors in the measurement of parameters characterizing the spin-induced effects. However, as mentioned earlier, for many parts of the parameter space, we find that errors are larger than $100 \%$ for which the quoted errors should be considered as "bounds" on the parameter in question.

Results and discussion. The dependences of the errors (for a fixed SNR of 10) in measuring $\kappa_{s}$ as a function of the total mass, for few mass ratio cases (top panel) and spin configurations for a near-equal mass system (bottom panel) for advanced LIGO sensitivity are shown in Fig. 1. This clearly shows that the proposed test works very well for highly spinning, near-equal mass systems. Evidently, the observed improvement for rapidly spinning systems can be attributed to the large spin-induced quadrupole moment they possess. In addition, for nearly equal mass systems, the best estimates of $\kappa_{s}$ come from compact binaries in which the spins of both components are aligned with respect to the orbital angular momentum vector of the binary, and the worst estimates are for 
those cases where the component spins are antialigned with respect to the orbital angular momentum. The decrease in the errors with mass ratio may be attributed to the additional mass ratio and inclination angle dependences that amplitude corrections bring in, which affect the correlation of $\kappa_{S}$ with other parameters (especially spins) in a nontrivial way leading to the observed trend. On the other hand, the dependence of the errors on the spin orientation is due to its effects on the upper cutoff frequency. The figure shows that even with a moderate SNR of 10, the proposed test works very well for a number of mass ratio and spin configurations, where the best cases have $\Delta \kappa_{s}<0.5(50 \%)$. It is worth recalling that the allowed values of $\kappa_{s}$ for BBH mimickers, such as binaries involving boson stars, can be as high as 150 . Hence, the expected bounds are capable of putting stringent constraints on those models.

Figure 2 displays the dependence of the errors of $\kappa_{s}$ on the component spins for two representative stellar mass compact binaries with component masses $(5,4) M_{\odot}$ and $(10,9) M_{\odot}$. The results are very promising and show that for dimensionless spins larger than 0.5 , the errors in estimating $\kappa_{s}$ are smaller than $\sim 5$ in both the cases. This would mean that the proposed test could be effective in certain cases even with moderate spins.

Since the GW detectors are poised to observe tens to hundreds of BBH mergers in the coming years, we also have the interesting possibility of combining the constraints from these individual observations. If there are $N$ detections, the errors go down by roughly a factor of $\sqrt{N}$. Hence, the combined posterior of about 100 events on the null hypothesis may narrow down the constraints on $\kappa_{s}$ by a factor of 10 .
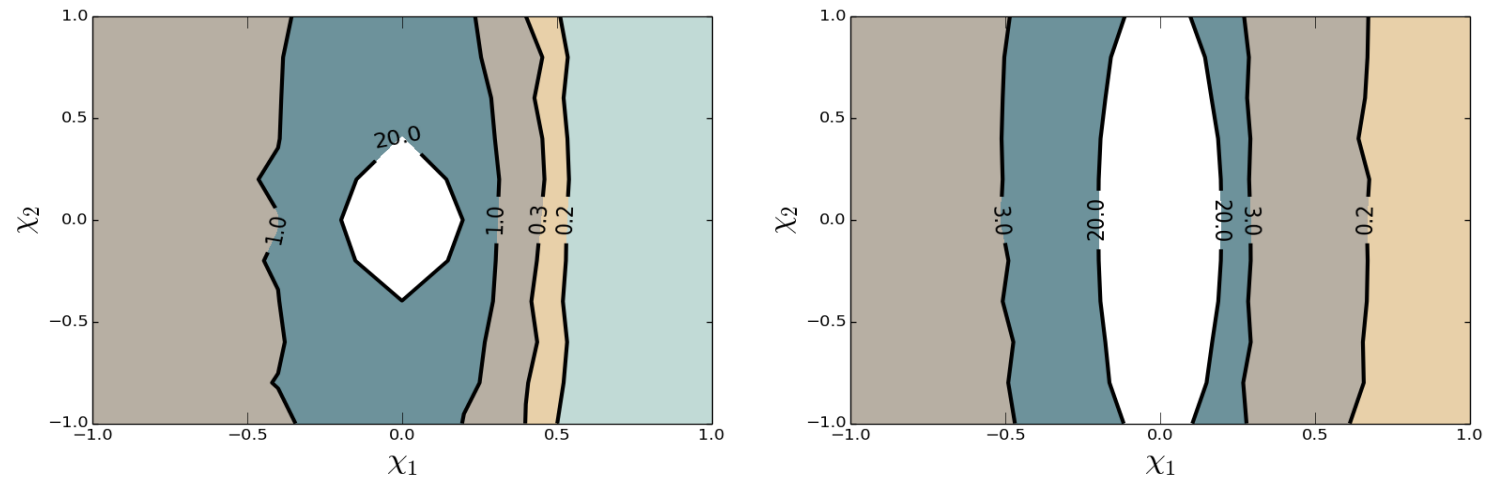

FIG. 3: Projected constraints from GW observations of SMBBH mergers by the LISA detector as a function of the component spins for two representative SMBBH configurations $\left(5 \times 10^{6}, 10^{6}\right) M_{\odot}$ (top panel) and $\left(10^{7}, 10^{6}\right) M_{\odot}$ (bottom panel) located at 3 Gpc. The inclination angle of the binary is chosen to a value of $\pi / 3$.

Possible constraints on $\kappa_{s}$ from space-based detectors. With the recent success of of LISA pathfinder mission [44], there is renewed interest in pursuing a GW detector in space with low frequency sensitivity capable of observing supermassive BBH (SMBBH) mergers. Towards this goal, we extend our study to the case of low frequency space-based detectors like LISA and projected constraints possible on $\kappa_{s}$ from them. The results are shown in Fig. 3 which uses the noise PSD of Ref. [45]. The SMBBH system is assumed to be at a luminosity distance of $3 \mathrm{Gpc}$. We find that the LISA observations of SMBBH mergers can very accurately constrain the $\kappa_{s}$ parameter and, hence, confirm the BBH nature of the observed sources, tightly constraining any alternatives to BBHs. It should be clear from Fig. 3 that errors in measuring $\kappa_{s}$ are smaller than $10 \%$ for a number of configurations with moderate spins, making the test an extremely deep probe of any possible deviation from BBH nature. These results show how LISA can be a very sensitive probe of fundamental physics.

Possible constraints on BH mimickers. Since boson stars can have $\kappa$ between $\sim 10$ and 150 [28], binary systems of boson stars may have $\kappa_{s}$ in the range $\sim 10-150$. This allowed range lies well within the reach of the proposed test. Recently, for slowly rotating thin shell gravastars, Ref. [29] showed that the spin-induced quadrupole can take a wide range of values depending on the specifics of the model (see Fig. 7 of Ref. [29]). This range includes $\kappa=1$, the BH value, too. Indeed, if $\kappa_{\mathrm{GS}}=1$, our test will not be able to distinguish it from a BH. Except for this very fine-tuned scenario, the projected bounds from the proposed test might significantly help to constrain the allowed parameter space of gravastars and can influence the theoretical developments in the field. The details of the bounds possible on specific BH mimicker models will be reported elsewhere [46].

We note that the proposed test may not be very sensitive in distinguishing a BH from a BH mimicker in a NSBH system. This is because the neutron stars are expected to have small spins $(\leqslant 0.05)$ for which spin-induced quadrupole would be very small. Moreover, since NSs are expected to have $\kappa$ value in the range of $2-14$, very accurate estimation of the $\kappa$ parameters of both the binary components is necessary to make the above distinction. This may be possible only with the future generation of GW detectors. 
There are some effects which can potentially contaminate the effectiveness of the proposed test. Because the compact objects in binaries are, strictly speaking, not isolated, the no-hair conjecture holds only approximately due to which there can be systematic effects which may affect the test (see Ref. [47] for a discussion on this aspect). Further, if the BHs are charged, then the resulting values of $\kappa$ will be offset from the Kerr value. Lastly, the choice of upper cutoff frequency may be different from ours if the object has structure and, hence, can cause systematic errors in our estimates. These issues need more careful examination which will be carried out in the future.

We conclude by noting that once implemented in a Bayesian framework, this proposal can be used to represent every detected compact binary system as contours in the $\kappa_{1}-\kappa_{2}$ space. Using multiple observations, the joint posteriors can tighten the bounds from this proposed null test, potentially constraining the parameter space allowed for non-BH compact objects. Inclusion of precessional features in the waveform and incorporating this effect into effective one body waveforms or phenomenological waveforms, which capture merger and ringdown phases as well, are likely to yield tighter constraints and will be explored in the future.

Acknowledgement. K. G. A. and N. V. K. were partially supported by a grant from Infosys Foundation. K. G. A. acknowledges the support by the Indo-US Science and Technology Forum through the Indo-US Centre for the Exploration of Extreme Gravity (Grant No. IUSSTF/JC-029/2016). This work was initiated following a suggestion by Luc Blanchet. We thank N. J.-McDaniel for very useful discussions on many aspects, especially those related to boson stars and gravastars. We thank Walter Del Pozzo for useful comments on the manuscript. We are thankful to P. Ajith, G. Faye, B. R. Iyer, S. Kastha, A. Laddha, and B. S. Sathayprakash for very useful discussions. This research has significantly benefitted from the interactions during the "Future of Gravitational Wave Astronomy Workshop" at the International Centre for Theoretical Sciences (Code No. ICTS/Prog-fgwa/2016/04). This document has LIGO preprint number P1600340.

\section{SUPPLEMENTAL MATERIAL}

\section{Spin-induced quadrupole and octupole pieces in compact binary waveforms}

The waveform used in this work is a variant of the one that is presented in Ref. [35]. These are constructed by simply making the dependences on parameters characterising the spin-induced quadrupole moment (through $\kappa_{s}$ and $\kappa_{a}$ ) and spin-induced octupole moment (through $\lambda_{s}$ and $\lambda_{a}$ ) explicit in the waveform, which were set to their respective values for Kerr BHs while writing the waveform model of Ref. [35]. In this note we list various pieces of the waveform where such dependences occur.

Let us first recall the schematic expression for the frequency domain amplitude of a gravitational wave signal, $\tilde{h}(f)$, given in Ref. [35]. ${ }^{1}$ This reads

$$
\tilde{h}(f)=\frac{M^{2}}{D_{L}} \sqrt{\frac{5 \pi}{48 \eta}} \sum_{n=0}^{4} \sum_{k=1}^{6} V_{k}^{n-7 / 2} C_{k}^{(n)} e^{\mathrm{i}\left(k \Psi_{\mathrm{SPA}}(f / k)-\pi / 4\right)} .
$$

Here, $M, \eta$ and $D_{L}$ denote the total mass, symmetric mass ratio parameter and the distance to the binary, respectively and the indices $n$ and $k$ denote the PN order and harmonic number, respectively. The coefficients $C_{k}^{(n)}$ denote the amplitude corrections associated with the contribution from $k$ th harmonic at $n$th PN order. Related expressions for each of the $C_{k}^{(n)}$ s can be found in Ref. [33, 35]. Here we list the only coefficient which has explicit dependence on the parameters $\left(\kappa_{s}\right.$ and $\left.\kappa_{a}\right)$ and corresponds to the contributions from the 2 nd harmonic at the $2 \mathrm{PN}$ order $\left(C_{2}^{(4)}\right)$. In addition, $\Psi_{\text {SPA }}$ represents the phase of the first harmonic in the frequency domain as obtained under the Stationary Phase Approximation (SPA) (see sec. VI of Ref. [33] for details on SPA). Schematically the expression for this phase can be written as follows

$$
\Psi_{\mathrm{SPA}}(f)=2 \pi f t_{\mathrm{c}}-\phi_{\mathrm{c}}+\left\{\frac{3}{128 \eta v^{5}}\left[\psi_{\mathrm{NS}}+\psi_{\mathrm{SO}}+\psi_{\mathrm{SS}}+\psi_{\mathrm{SSS}}\right]\right\}_{v=V_{1}(f)},
$$

where $\phi_{\mathrm{c}}$ denotes the orbital phase at the instant $t_{\mathrm{c}}$ of coalescence.

\footnotetext{
${ }^{1}$ Pre-factor of Eq. 1 of Ref. [35] should be multiplied with a factor $1 / \sqrt{\eta}$. We have corrected this in the Eq. (0.1).
} 
Further, one can write the spin part of the SPA phase more explicitly as

$$
\psi_{\text {Spin }} \equiv \psi_{\text {SO }}+\psi_{\text {SS }}+\psi_{\text {SSS }}=v^{3}\left[\mathcal{P}_{3}+\mathcal{P}_{4} v+\mathcal{P}_{5} v^{2}+\mathcal{P}_{6} v^{3}+\mathcal{P}_{7} v^{4}+\mathcal{P}_{8} v^{5}+\cdots\right]
$$

Again expressions for the coefficients $\mathcal{P}_{n}$ can be found in Ref. [33, 35] where explicit dependence on $\kappa_{s}$ and $\kappa_{a}$ is suppressed by setting them to their respective values for Kerr BBHs. Here we provide expressions for coefficients that contain explicit dependence on $\kappa_{s}$ and $\kappa_{a}$. Below we list the amplitude/phase coefficients that do contain explicit dependence on $\kappa_{s}$ and $\kappa_{a}$ and can be combined to those listed in Ref. [33,35] to write the final waveform expression.

$$
\begin{aligned}
C_{2}^{(4)} & =\frac{1}{\sqrt{2}}\left\{F _ { + } \left[\frac{113419241}{40642560}+\frac{152987}{16128} \eta-\frac{11099}{1152} \eta^{2}+\left(\frac{165194153}{40642560}-\frac{149}{1792} \eta+\frac{6709}{1152} \eta^{2}\right) c_{\iota}^{2}+\left(\frac{1693}{2016}-\frac{5723}{2016} \eta+\frac{13}{12} \eta^{2}\right) c_{\iota}^{4}\right.\right. \\
& -\frac{1}{24}\left(1-5 \eta+5 \eta^{2}\right) c_{\iota}^{6}+\left(1+c_{\iota}^{2}\right)\left[\left(\chi_{\mathrm{s}} \cdot \hat{\boldsymbol{L}}_{\mathrm{N}}\right)^{2}\left(\frac{1}{32}+\frac{23 \eta}{8}+\frac{3 \delta \kappa_{a}}{2}+\frac{3}{2}(1-2 \eta) \kappa_{s}\right)+\left(\chi_{\mathrm{a}} \cdot \hat{\boldsymbol{L}}_{\mathrm{N}}\right)^{2}\left(\frac{1}{32}-3 \eta+\frac{3 \delta \kappa_{a}}{2}\right.\right. \\
& \left.\left.\left.+\frac{3}{2}(1-2 \eta) \kappa_{s}\right)+\left(\chi_{\mathrm{a}} \cdot \hat{\boldsymbol{L}}_{\mathrm{N}}\right)\left(\chi_{\mathrm{s}} \cdot \hat{\boldsymbol{L}}_{\mathrm{N}}\right)\left(\frac{\delta}{16}+3(1-2 \eta) \kappa_{a}+3 \delta \kappa_{s}\right)\right]\right]+\mathrm{i} c_{\iota} F_{\times}\left[\frac{114020009}{20321280}+\frac{133411}{8064} \eta-\frac{7499}{576} \eta^{2}\right. \\
& +\left(\chi_{\mathrm{s}} \cdot \hat{\boldsymbol{L}}_{\mathrm{N}}\right)^{2}\left(\frac{1}{16}+\frac{23}{4} \eta+3 \delta \kappa_{a}+3(1-2 \eta) \kappa_{s}\right)+\left(\chi_{\mathrm{a}} \cdot \hat{\boldsymbol{L}}_{\mathrm{N}}\right)^{2}\left(\frac{1}{16}-6 \eta+3 \delta \kappa_{a}+3(1-2 \eta) \kappa_{s}\right)+\left(\chi_{\mathrm{a}} \cdot \hat{\boldsymbol{L}}_{\mathrm{N}}\right)\left(\chi_{\mathrm{s}} \cdot \hat{\boldsymbol{L}}_{\mathrm{N}}\right)\left(\frac{\delta}{8}\right. \\
& \left.\left.\left.+6(1-2 \eta) \kappa_{a}+6 \delta \kappa_{s}\right)+\left(\frac{5777}{2520}-\frac{5555}{504} \eta+\frac{34}{3} \eta^{2}\right) c_{\iota}^{2}-\frac{1}{4}\left(1-5 \eta+5 \eta^{2}\right) c_{\iota}^{4}\right]\right\} \Theta\left(2 F_{\mathrm{cut}}-f\right)
\end{aligned}
$$

$$
\begin{aligned}
\mathcal{P}_{4} & =-\frac{5}{8}\left(\chi_{\mathrm{s}} \cdot \hat{\boldsymbol{L}}_{\mathrm{N}}\right)^{2}\left[1+156 \eta+80 \delta \kappa_{a}+80(1-2 \eta) \kappa_{s}\right]+\left(\chi_{\mathrm{a}} \cdot \hat{\boldsymbol{L}}_{\mathrm{N}}\right)^{2}\left[-\frac{5}{8}-50 \delta \kappa_{a}-50 \kappa_{s}+100 \eta\left(1+\kappa_{s}\right)\right] \\
& -\frac{5}{4}\left(\chi_{\mathrm{a}} \cdot \hat{\boldsymbol{L}}_{\mathrm{N}}\right)\left(\chi_{\mathrm{s}} \cdot \hat{\boldsymbol{L}}_{\mathrm{N}}\right)\left[\delta+80(1-2 \eta) \kappa_{a}+80 \delta \kappa_{s}\right], \\
\mathcal{P}_{6} & =\pi\left[\frac{2270}{3} \delta \chi_{\mathrm{a}} \cdot \hat{\boldsymbol{L}}_{\mathrm{N}}+\left(\frac{2270}{3}-520 \eta\right) \chi_{\mathrm{s}} \cdot \hat{\boldsymbol{L}}_{\mathrm{N}}\right]+\left(\chi_{\mathrm{s}} \cdot \hat{\boldsymbol{L}}_{\mathrm{N}}\right)^{2}\left[-\frac{1344475}{2016}+\frac{829705}{504} \eta+\frac{3415}{9} \eta^{2}+\delta\left(\frac{26015}{28}-\frac{1495}{6} \eta\right) \kappa_{a}\right. \\
& \left.+\left(\frac{26015}{28}-\frac{44255}{21} \eta-240 \eta^{2}\right) \kappa_{s}\right]+\left(\chi_{\mathrm{a}} \cdot \hat{\boldsymbol{L}}_{\mathrm{N}}\right)^{2}\left[-\frac{1344475}{2016}+\frac{267815}{252} \eta-240 \eta^{2}+\delta\left(\frac{26015}{28}-\frac{1495}{6} \eta\right) \kappa_{a}+\left(\frac{26015}{28}\right.\right. \\
& \left.\left.-\frac{44255}{21} \eta-240 \eta^{2}\right) \kappa_{s}\right]+\left(\chi_{\mathrm{a}} \cdot \hat{\boldsymbol{L}}_{\mathrm{N}}\right)\left(\chi_{\mathrm{s}} \cdot \hat{\boldsymbol{L}}_{\mathrm{N}}\right)\left[\left(\frac{26015}{14}-\frac{88510}{21} \eta-480 \eta^{2}\right) \kappa_{a}+\delta\left[-\frac{1344475}{1008}+\frac{745}{18} \eta+\left(\frac{26015}{14}\right.\right.\right. \\
& \left.\left.\left.-\frac{1495}{3} \eta\right) \kappa_{s}\right]\right], \\
\mathcal{P}_{7} & =\delta \chi_{\mathrm{a}} \cdot \hat{\boldsymbol{L}}_{\mathrm{N}}\left(-\frac{25150083775}{3048192}+\frac{26804935}{6048} \eta-\frac{1985}{48} \eta^{2}\right)+\chi_{\mathrm{s}} \cdot \hat{\boldsymbol{L}}_{\mathrm{N}}\left(-\frac{25150083775}{3048192}+\frac{10566655595}{762048} \eta-\frac{1042165}{3024} \eta^{2}\right.
\end{aligned}
$$$$
\left.+\frac{5345}{36} \eta^{3}\right)+\left(\chi_{\mathrm{s}} \cdot \hat{\boldsymbol{L}}_{\mathrm{N}}\right)^{3}\left[\frac{265}{24}+\frac{4035 \eta}{2}-\frac{20 \eta^{2}}{3}+\left(\frac{3110}{3}-\frac{10250}{3} \eta+40 \eta^{2}\right) \kappa_{s}+\delta\left[\left(\frac{3110}{3}-\frac{4030 \eta}{3}\right) \kappa_{a}\right.\right.
$$$$
\left.\left.-440(1-\eta) \lambda_{a}\right]-440(1-3 \eta) \lambda_{s}\right]+\left(\chi_{\mathrm{a}} \cdot \hat{\boldsymbol{L}}_{\mathrm{N}}\right)^{3}\left[\left(\frac{3110}{3}-\frac{8470}{3} \eta\right) \kappa_{a}-440(1-3 \eta) \lambda_{a}+\delta\left[\frac{265}{24}-2070 \eta\right.\right.
$$$$
\left.\left.+\left(\frac{3110}{3}-750 \eta\right) \kappa_{s}-440(1-\eta) \lambda_{s}\right]\right]+\left(\chi_{\mathrm{s}} \cdot \hat{\boldsymbol{L}}_{\mathrm{N}}\right)^{2}\left(\chi_{\mathrm{a}} \cdot \hat{\boldsymbol{L}}_{\mathrm{N}}\right)\left[\left(3110-\frac{28970}{3} \eta+80 \eta^{2}\right) \kappa_{a}-1320(1-3 \eta) \lambda_{a}\right.
$$$$
\left.+\delta\left[\frac{265}{8}+\frac{12055}{6} \eta+\left(3110-\frac{10310}{3} \eta\right) \kappa_{s}-1320(1-\eta) \lambda_{s}\right]\right]+\left(\chi_{\mathrm{a}} \cdot \hat{\boldsymbol{L}}_{\mathrm{N}}\right)^{2}\left(\chi_{\mathrm{s}} \cdot \hat{\boldsymbol{L}}_{\mathrm{N}}\right)\left[\frac{265}{8}-\frac{6500}{3} \eta+40 \eta^{2}\right.
$$$$
\left.+\left(3110-\frac{27190 \eta}{3}+40 \eta^{2}\right) \kappa_{s}+\delta\left[\left(3110-\frac{8530 \eta}{3}\right) \kappa_{a}-1320(1-\eta) \lambda_{a}\right]-1320(1-3 \eta) \lambda_{s}\right] .
$$

* Electronic address: krishnendu@cmi.ac.in

$\dagger$ Electronic address: kgarun@cmi.ac.in 
\#lectronic address: ckm@iitm.ac.in

[1] J. Aasi et al. (LIGO Scientific), Class. Quant. Grav. 32, 074001 (2015), arXiv:1411.4547.

[2] B. P. Abbott et al. (Virgo, LIGO Scientific), Phys. Rev. Lett. 116, 061102 (2016), arXiv:1602.03837.

[3] B. P. Abbott et al. (Virgo, LIGO Scientific), Phys. Rev. Lett. 116, 241103 (2016), arXiv:1606.04855.

[4] B. P. Abbott et al. (Virgo, LIGO Scientific), Phys. Rev. X6, 041015 (2016), arXiv:1606.04856.

[5] L. Blanchet, Living Rev. Rel. 9, 4 (2006), arXiv:1310.1528.

[6] M. Sasaki and H. Tagoshi, Living Rev. Rel. 6, 6 (2003), gr-qc/0306120.

[7] F. Pretorius (2007), relativistic Objects in Compact Binaries: From Birth to Coalescence Editor: Colpi et al., arXiv:0710.1338.

[8] B. P. Abbott et al. (Virgo, LIGO Scientific), Phys. Rev. Lett. 116, 221101 (2016), arXiv:1602.03841.

[9] R. P. Kerr, Phys. Rev. Lett. 11, 237 (1963).

[10] F. Acernese et al. (VIRGO), Class. Quant. Grav. 32, 024001 (2015), arXiv:1408.3978.

[11] B. Iyer et al., LIGO India Technical Document https://dcc.ligo.org/LIGO-M1100296/public (2011).

[12] B. P. Abbott et al. (Virgo, LIGO Scientific), Astrophys. J. 833, L1 (2016), arXiv:1602.03842.

[13] P. O. Mazur and E. Mottola, Proc. Nat. Acad. Sci. 101, 9545 (2004), gr-qc/0407075.

[14] M. Colpi, S. L. Shapiro, and I. Wasserman, Phys. Rev. Lett. 57, 2485 (1986).

[15] G. F. Giudice, M. McCullough, and A. Urbano, JCAP 1610, 001 (2016), arXiv:1605.01209.

[16] O. Dreyer et al., Class. Quantum Grav. 21, 787 (2004), gr-qc/0309007.

[17] E. Berti, V. Cardoso, and C. M. Will, Phys. Rev. D 73, 064030 (2006), gr-qc/0512160.

[18] E. Berti and V. Cardoso, Int. J. Mod. Phys. D15, 2209 (2006), gr-qc/0605101.

[19] C. F. B. Macedo, P. Pani, V. Cardoso, and L. C. B. Crispino, Phys. Rev. D88, 064046 (2013), arXiv:1307.4812.

[20] F. Ryan, Phys. Rev. D 56, 1845 (1997).

[21] D. Brown et al., Phys. Rev. Lett. 99, 201102 (2007).

[22] S. A. Hughes, Phys. Rev. D64, 064004 (2001).

[23] V. Cardoso, E. Franzin, A. Maselli, P. Pani, and G. Raposo (2017), arXiv:1701.01116.

[24] A. Maselli, P. Pani, V. Cardoso, T. Abdelsalhin, L. Gualtieri, and V. Ferrari (2017), arXiv:1703.10612.

[25] N. Sennett, T. Hinderer, J. Steinhoff, A. Buonanno, and S. Ossokine (2017), arXiv:1704.08651.

[26] W. G. Laarakkers and E. Poisson, Astrophys. J. 512, 282 (1999), gr-qc/9709033.

[27] G. Pappas and T. A. Apostolatos, Phys. Rev. Lett. 108, 231104 (2012), arXiv:1201.6067.

[28] F. D. Ryan, Phys. Rev. D55, 6081 (1997).

[29] N. Uchikata and S. Yoshida, Class. Quant. Grav. 33, 025005 (2016), arXiv:1506.06485.

[30] E. Poisson, Phys. Rev. D57, 5287 (1998), gr-qc/9709032.

[31] S. Marsat, Class. Quant. Grav. 32, 085008 (2015), arXiv:1411.4118.

[32] A. Buonanno, G. Faye, and T. Hinderer, Phys.Rev. D87, 044009 (2013), arXiv:1209.6349.

[33] K. G. Arun, A. Buonanno, G. Faye, and E. Ochsner, Phys. Rev. D79, 104023 (2009), arXiv:0810.5336.

[34] A. Bohé, G. Faye, S. Marsat, and E. K. Porter, Class. Quant. Grav. 32, 195010 (2015), arXiv:1501.01529.

[35] C. K. Mishra, A. Kela, K. G. Arun, and G. Faye, Phys. Rev. D93, 084054 (2016), arXiv:1601.05588.

[36] C. Cutler and E. Flanagan, Phys. Rev. D49, 2658 (1994).

[37] M. Vallisneri, Phys. Rev. D77, 042001 (2008), gr-qc/0703086.

[38] C. Van Den Broeck and A. S. Sengupta, Class. Quantum Grav. 24, 1089 (2007), gr-qc/0610126.

[39] K. G. Arun, B. R. Iyer, B. S. Sathyaprakash, S. Sinha, and C. Van Den Broeck, Phys. Rev. D 76, 104016 (2007), arXiv:0707.3920.

[40] S. Husa et al., Phys. Rev. D93, 044006 (2016), arXiv:1508.07250.

[41] M. Favata, K. G. Arun, C. Kim, J. Kim, and H. W.Lee (In preparation).

[42] R. Balasubramanian and S. V. Dhurandhar, Phys. Rev. D57, 3408 (1998).

[43] P. Ajith, Phys.Rev. D84, 084037 (2011), arXiv:1107.1267.

[44] M. Armano et al., Phys. Rev. Lett. 116, 231101 (2016).

[45] S. Babak, J. Gair, A. Sesana, E. Barausse, C. F. Sopuerta, C. P. L. Berry, E. Berti, P. Amaro-Seoane, A. Petiteau, and A. Klein, (2017), arXiv:1703.09722.

[46] N. V. Krishnendu et al. (In preparation).

[47] M. Campanelli, C. O. Lousto, and Y. Zlochower, Phys. Rev. D79, 084012 (2009), arXiv:0811.3006. 